\title{
Does NPWT improve the outcome of osteomyelitis in HCV-HIV coinfected patient?
}

\author{
Ciro Pempinello, Alfredo Franco, Fiorella Martucci
}

\section{CASE REPORT}

\begin{abstract}
Osteomyelitis with severe skin and soft tissue damage in critically ill patients cannot be treated solely by an orthopaedic specialist. In such patients, a team approach is required. We report the treatment of osteomyelitis in a $\mathrm{HCV}$ and HIV positive patient with several severe comorbidities (liver failure, peripheral vascular disease and peripheral neuropathy). The patient underwent mechanical debridement, followed by a three months of antibiotic therapy. Six months later the infection relapsed and a second debridement was performed. V.A.C.Ulta therapy with instillation of "Dermacyn" solution, a super-oxidized disinfectant, as antiseptic solution was applied for 20 days and the wound healed. Three-years follow-up with inflammatory laboratory tests, clinical and radiographic examinations showed healing of the wound without any relapse of infection. In this paper, we suggest combining debridement and NPWT with instillation for critical patients with severe soft tissue damage that poorly respond to other therapies.
\end{abstract}

Keywords-antibiotic therapy, HCV, HIV, osteomyelitis, Negative Pressure Wound Therapy, instillation

\section{INTRODUCTION}

$\mathbf{P}$ STEOMYELITIS is an heterogeneous disease encompassing a spectrum of severity grades with different therapeutic requirements that necessitate long-term follow-up. Joint infections and osteomyelitis, especially involving foreign implants, are difficult to treat and require prolonged antibiotic therapy! 12 Therefore, the importance of antimicrobial therapy in the treatment of osteomyelitis is unquestioned. ${ }^{3}$ Surgical debridement is also a cornerstone in the treatment of patients with osteomyelitis and removal of infected bone unquestionably improves the chance of a successful outcome.

A male 44 year-old patient, born in Ukraine, HCV/HIV coinfected with compromised immune response presented relapse of knee infection with fistula, probably influenced by the individual conditions of the host. ${ }^{4}$ Thus, the appropriate therapeutic strategy in this case included aggressive surgical debridement and vacuum assisted closure (VAC) therapy with instillation of antiseptic solution.

Manuscript received 21.10.2016; revised 02.11.2016. This work did not receive any financial support. Authors declare no conflict of interest.

Author affiliations: Department of Orthopedic and Traumatology - S. Gennaro Hospital, Via S.Gennaro dei Poveri, 25 -80127- Napoli - Italy, (CP); Department of Infectious Diseases Cotugno Hospital, Via G. Quagliariello, 54 - 80131- Napoli - Italy , (AF, FM)

*Correspondence to: Ciro Pempinello, email: cpempinello@gmail.com, Alfredo Franco, email: alfredo.franco@ospedaledeicolli.it, Fiorella Martucci email: fiorella.martucci@ospedalideicolli.it

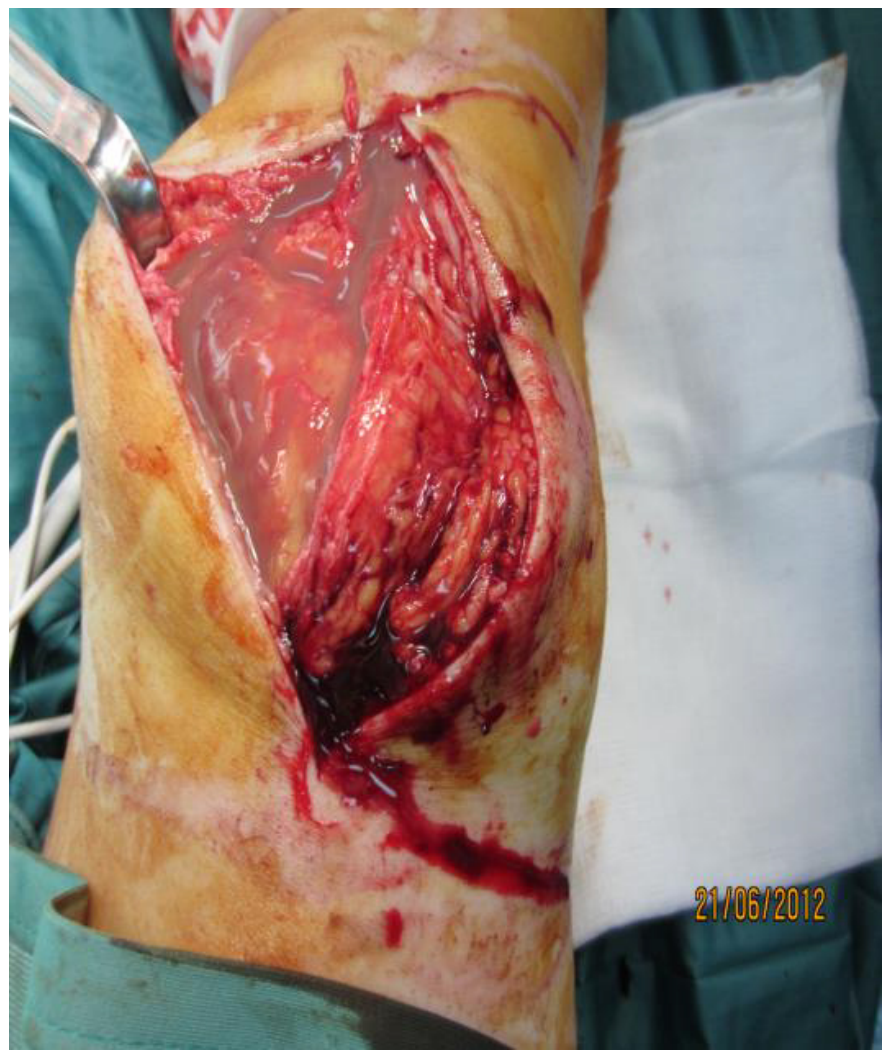

Figure 1. Initial wound debridement

\section{CASE STUDY}

In this paper, we report the case of a 44 year-old patient affected by severe osteomyelitis and HCV/HIV infection. He had a history of liver failure (HCV related hepatopathy) and immunodeficiency. The patient had abused alcohol and illicit drugs as well as heroine in the past but he denied using any illicit drugs over the last six months.

On July 2nd 2012, during the first in-patient treatment, the patient presented with severe sepsis and fever $\left(>39^{\circ} \mathrm{C}\right)$, severe resting knee pain, local inflammation with sinus tract and elevated laboratory values (CRP $80 \mathrm{mg} / \mathrm{l}$; lymphocyte count of $6000 / \mathrm{ml}$ ). The wound exudate culture showed positive for methicilin resistant Staphylococcus aureus (MRSA). The patient was HIV positive (HIV infection category A2 


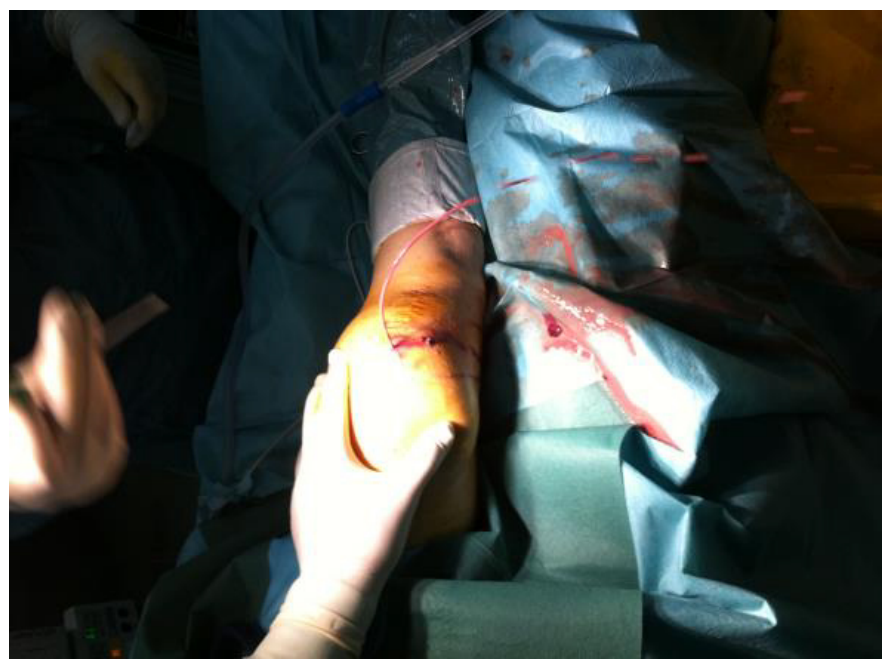

Figure 2. Reinfection of the wound 6 months after initial therapy

according to the CDC classification ${ }^{5}$ ) with $\mathrm{CD} 4$ count equal to 334 cells $/ \mu \mathrm{l}$ and $\mathrm{CD} 4 / \mathrm{CD} 8$ ratio of 0.64 . CD4 cell count is independent factor that affects wound healing in HIV/AIDS patient. HIV 1-Quantitative RNA was $186,045 \mathrm{cp} / \mathrm{ml}$ and the patient started receiving ATRIPLA (TDF+FTC+EFV) 1 tablet daily on February 5th 2013.

An antibiotic regime tailored to the sensitivities of the organism isolated from the obtained biopsy material was started prior to surgical operation. Nevertheless, during the operation a huge quantity of purulent material was discovered and aspirated. Radical debridement was performed (Fig. II).

The patient was treated with a combination of $160 \mathrm{mg}$ sulfamethoxazole and $800 \mathrm{mg}$ trimethoprim (TMP/SMZ) and rifampicin $(10 \mathrm{mg} / \mathrm{kg}$ body weight) orally for 3 months and application of silver-coated dressings as bacterial barriers (ACTICOAT, Smith \& Nephew).

Six months later the infection relapsed with sinus tract involvement and acute symptoms (Fig 2) and the patient was reoperated and wound debridement was performed (Fig 3 ). The skin was left open and V.A.C.Ulta Negative Pressure Wound Therapy system was applied (pressure $-125 \mathrm{mmHg}$ ) with instillation of "Dermacyn" solution, a super-oxidized disinfectant, as antiseptic solution. Dressing changes were performed every two days. The V.A.C.Ulta was applied, for 20 days (Fig. 4). Skin closure was performed in two stages: first the subcutaneous tissue and later on the skin. Three years later, the patient had no signs of infection, rigid knee with limited extension $\left(<170^{\circ}\right)$ and flexion $\left(<40^{\circ}\right)$, limb shortening $(<3$ $\mathrm{cm})$, normal laboratory values, and was able to walk without crutches or brace (Fig. 5).

\section{DISCUSSION}

Acute and chronic osteomyelitis, spondylodiscitis, and orthopedic implant infections are a heterogeneous group of infectious disease entities. One common denominator is the complex diagnostic and therapeutic challenges that are regularly encountered by clinicians. The patient's history, subjective symptoms, and biochemical and physical findings are often inconclusive, particularly in the early disease stages.

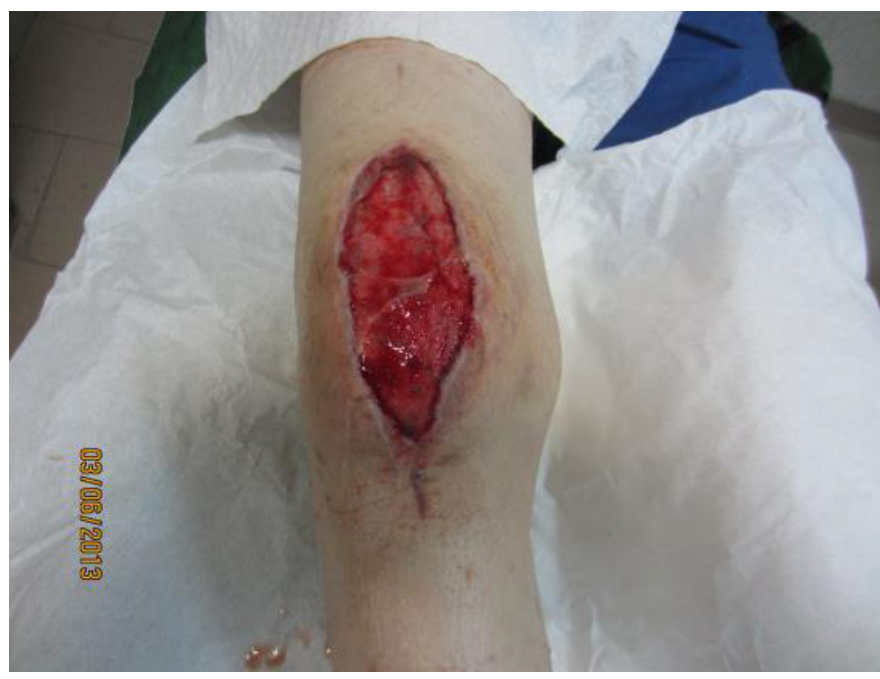

Figure 3. Second wound debridement

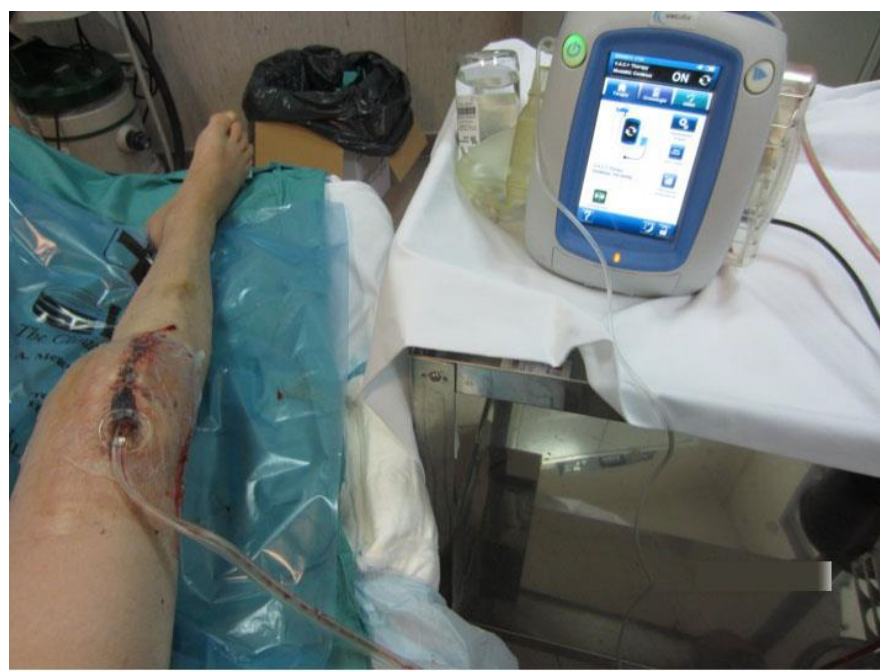

Figure 4. V.A.C.Ulta Therapy with instillation

After years in which planar scintigraphy used to be the standard in the diagnosis of osteomyelitis, the need for improved localization was recently met by single-photon emission computed tomography (SPECT) with 111In-WBC combined with 99m Tc-MDP or 99mTc-sulfur colloid and positron emission tomography with fludeoxyglucose (FDG/PET) ${ }^{6}$ However, SPECT and FDG/PET are expensive for the health care system and have showed low specificity for periprosthetic infection. Osteomyelitis still presents problems in both diagnostic procedures and in treatment. When osteomyelitis is associated with a foreign body or orthopedic device, it is common for bacterial pathogens to persist despite antibiotic therapy, and the infection is often cured only after removal of the implant and debridement of necrotic bone.$^{7}$ Radical debridement is a prerequisite for cure in any orthopedic infection, as debridement will remove the largest bio-burden, but even the most careful cleaning cannot prevent residual small bacterial colonies from forming new habitats in niches of the debrided site. Surgical debridement is the cornerstone in the treatment 
of patients with osteomyelitis $\frac{8}{}$ All isolated pockets of granulation tissue of necrosis must be sampled. Cleaning is finalized using intense pulsatile lavage with saline combined with an antiseptic solution to make sure to remove all necrotic tissue in microscopic areas. An antibiotic susceptibility testing of the organisms isolated from the obtained biopsy material should be started after surgery ${ }^{[9}$ Multiple previous studies have noted that diabetes, obesity, and liver failure predispose patients to a number of complications, including wound infections, showing that host compromised immune system may play a crucial role in the outcome of the disease $\frac{10}{10}$

Early and aggressive surgical treatment for these critical patients is not sufficient to heal the infection and leads to poor patient outcomes. Sometimes the infected foci are difficult to eradicate and modified antibiotic therapy is necessary after considering several factors, such as bacteremia, clinical findings, serial debridement isolates and their sensitivities, inadequate serum bactericidal levels, abnormal laboratory studies, and/or patient intolerances!11 Here, we describe the clinical course of a critical patient with HCV/HIV coinfection and immune deficiency who developed a severe knee infection. In this patient scintigraphy was not performed. X-ray examination showed bone necrosis. The other diagnostic procedures were not performed and were considered useless due to the severe clinical picture with high fever, skin redness and acute knee pain.

This critical patient was treated with multiple approaches, as well as combinations of debridement and VAC therapy with instillation of antiseptic solution that are not considered standard care or current orthopedic practice. This patient presented with acute illness, so difficult-to-heal wounds were successfully treated with application of V.A.C.Ulta. ${ }^{[4}$ The V.A.C.Ulta Therapy System (KCI/Acelity USA) is an integrated wound therapy system that provides Negative Pressure Therapy (NPT) with an instillation option. V.A.C.Ulta Therapy consists of VAC therapy coupled with automated, controlled delivery and removal of topical wound solutions in the wound bed. This patient HCV/HIV coinfected with compromised immune surveillance showed acute illness, relapse of infection, and difficult wound healing that was later on successfully treated by application of V.A.C.Ulta. Three-years follow-up after care shows complete healing of wounds, moderate knee pain, without any relapse of infection and the patient walks under arm crutches at home. Therefore, we suggest considering different therapeutic strategies for patients with severe skin and soft tissue damage and comorbidities. In our opinion, critical patients affected by severe comorbidities could be treated using non-standard procedures as well as negative pressure wound therapy with instillation. However, it has to be considered that wounds with osteomyelitis could be treated with NPWT after radical debridement and initiation of appropriate antibiotic therapy. In other case it is contraindicated.

The clinical conditions of the patient showed fairly satisfactory outcomes at follow-up over 3 years. Accordingly, the results of this study demonstrates the constant evolution of surgical techniques, and that the combination of various approaches with non-standard procedures can give good clinical outcomes, even though they are only applicable to a minority

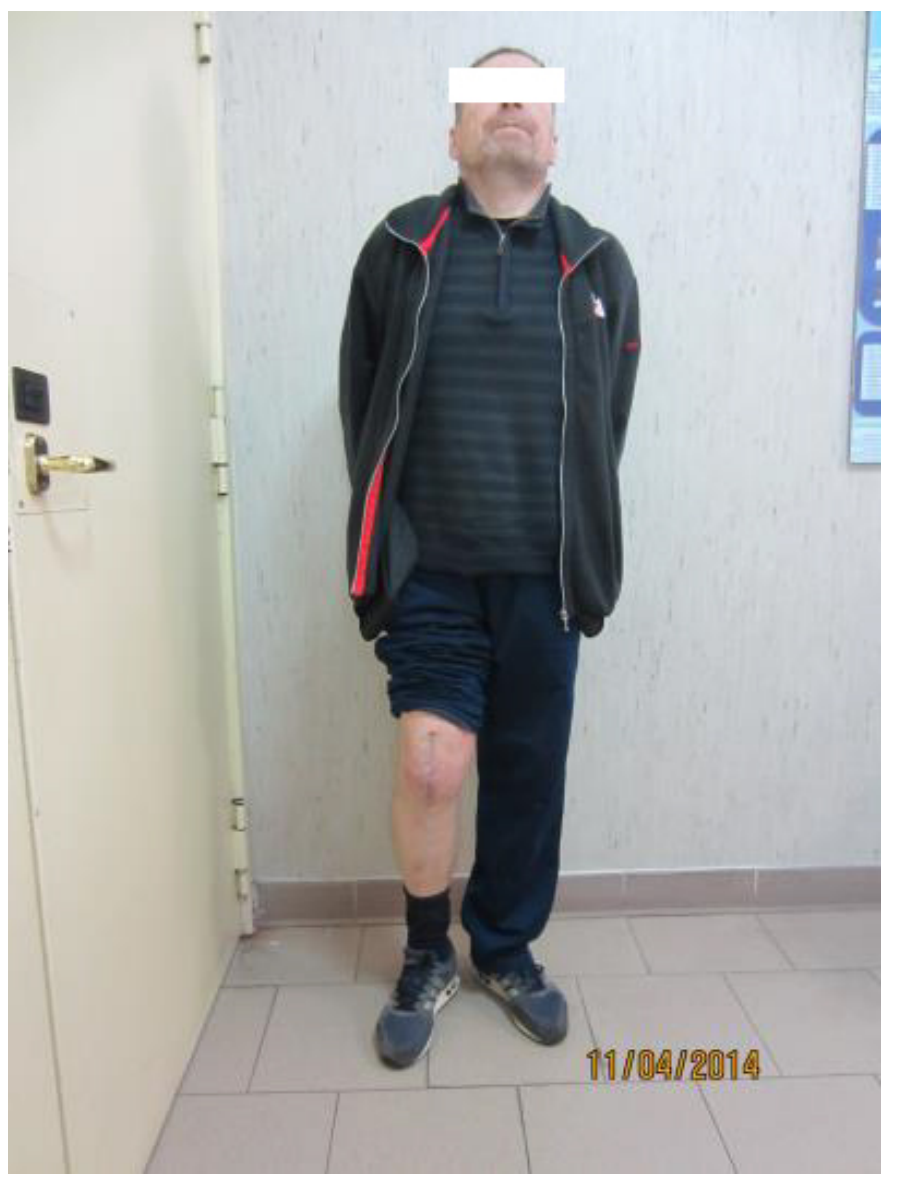

Figure 5. Full weight-bearing leg wit a healed wound

of most severely ill patients.

\section{REFERENCES}

[1] B. Spellberg and B. A. Lipsky, "Systemic antibiotic therapy for chronic osteomyelitis in adults," Clinical Infectious Diseases, vol. 54, no. 3, pp. 393-407, dec 2011. [Online]. Available: https://doi.org/10.1093\%2Fcid\%2Fcir842

[2] W. Zimmerli, A. Trampuz, and P. E. Ochsner, "Prosthetic-joint infections," New England Journal of Medicine, vol. 351, no. 16, pp. 1645-1654, oct 2004. [Online]. Available: https://doi.org/10.1056\% 2Fnejmra040181

[3] T. Ascione, M. Iannece, P. Rosario, R. Pempinello, P. Pagliano, M. Conte, C. Pempinello, R. Rotondo, M. Mariconda, and S. Lepore, "Impact of therapeutic choices on outcome of osteomyelitis caused by MRSA," Journal of Infection, vol. 63, no. 1, pp. 102-104, jul 2011. [Online]. Available: https://doi.org/10.1016\%2Fj.jinf.2011.04.015

[4] B. Lehner, W. Fleischmann, R. Becker, and G. N. Jukema, "First experiences with negative pressure wound therapy and instillation in the treatment of infected orthopaedic implants: a clinical observational study," International Orthopaedics, vol. 35, no. 9, pp. 1415-1420, may 2011. [Online]. Available: https: //doi.org/10.1007\%2Fs00264-011-1274-y

[5] K. G. Castro, J. W. Ward, L. Slutsker, J. W. Buehler, H. W. Jaffe, R. L. Berkelman, and J. W. Curran, "1993 revised classification system for hiv infection and expanded surveillance case definition for aids among adolescents and adults," MMWR Recomm Rep, vol. 41, no. RR-17, pp. 1-19, 1992.

[6] W. van der Bruggen, C. P. Bleeker-Rovers, O. C. Boerman, M. Gotthardt, and W. J. Oyen, "Pet and spect in osteomyelitis and prosthetic bone and joint infections: a systematic review," in Seminars in nuclear medicine, vol. 40, no. 1. Elsevier, 2010, pp. 3-15.

[7] C. Pempinello, T. Ascione, M. Conte, A. Bova, and R. Pempinello, "Periprosthetic infections in critical patients: a challenge for orthopaedics," Journal of Orthopaedic Trauma, vol. 12, p. S61, 2011. 
[8] G. Cierny, J. T. Mader, and J. J. Penninck, "The classic: A clinical staging system for adult osteomyelitis," Clinical Orthopaedics and Related Research, vol. 414, pp. 7-24, sep 2003. [Online]. Available: https://doi.org/10.1097\%2F01.blo.0000088564.81746.62

[9] A. John, D. Baldoni, M. Haschke, K. Rentsch, P. Schaerli, W. Zimmerli, and A. Trampuz, "Efficacy of daptomycin in implantassociated infection due to methicillin-resistant staphylococcus aureus: Importance of combination with rifampin," Antimicrobial Agents and Chemotherapy, vol. 53, no. 7, pp. 2719-2724, apr 2009. [Online].
Available: https://doi.org/10.1128\%2Faac.00047-09

[10] J. Ciampolini, "Pathophysiology of chronic bacterial osteomyelitis. why do antibiotics fail so often?" Postgraduate Medical Journal, vol. 76, no. 898, pp. 479-483, aug 2000. [Online]. Available: https://doi.org/10.1136\%2Fpmj.76.898.479

[11] C. Pempinello, A. Bova, and R. Pempinello, "A case of severe periprosthetic infection of the knee," Giornale Italiano di Ortopedia e Traumatologia, vol. 39, pp. 16-20, 2013 